\title{
Mathis Heinrich
}

\section{Zwischen Bankenrettungen und autoritärem Wettbewerbsregime Zur Dynamik des europäischen Krisenmanagements ${ }^{1}$}

\section{Einleitung}

Die Weltfinanzkrise und die begleitenden Krisen haben die Europäische Union (EU) nicht nur in eine langanhaltende Rezession gestürzt, sondern auch tiefgreifende Widersprüche in der Organisation und Regulation der europäischen Ökonomie offenbart. Gleichzeitig erscheint - zumindest zu Beginn der Krise - der Glaube in die perfekte Funktionsweise der (Finanz-) Märkte stark erschüttert. Aber haben sich die Organisationsmuster und Regulationsmodi der europäischen Kapitalismusformen sowie die ideologischen und diskursiven Denk- und Interpretationsmuster der europäischen Integrationsweise im Zuge der Krise tatsächlich entscheidend transformiert? Dies kann heute stark bestritten werden. Die Transformationsprozesse im Zuge der Krise, so die wesentliche These dieses Beitrags, sind eher als bescheiden zu bewerten, bzw. weisen partiell sogar in die falsche Richtung. Das europäische Krisenmanagement bildet damit vielmehr eine Verschärfung bestehender transnationaler und diskursiver Politikund Integrationsdynamiken, durch die nicht nur die bestehenden europäischen Machtnetzwerke und Kompromissstrukturen transformativ reproduziert werden, sondern auch eine fortdauernde Desartikulation und -Organisation möglicher Alternativen bestärkt wird.

Die strukturellen Ursachen der Weltfinanzkrise sind zweifelsohne vielschichtig, und gehen im Wesentlichen auf die Reaktionsweise auf die Krisen- und Stagnationsphase der 1970er Jahre zurück, indem eine wachsende Globalisierung und Realisierung nationaler Akkumulationsstrategien über die Expansion und Liberalisierung der Finanzmärkte es ermöglichten, die entstandene Kaufkraftlü-

1 Die hier aufgeführten Ergebnisse und Überlegungen beruhen auf einer zusammen mit Hans-Jürgen Bieling erarbeiteten Studie zur Transformation des europäischen Finanzsystems für das Institut für Gesellschaftsanalyse der Rosa-Luxemburg Stiftung (siehe Bieling/Heinrich 2012). 
cke durch private Verschuldung auszugleichen und neue finanzmarktvermittelte Investitions- und Profitoptionen zu schaffen (Harvey 2003). Gleichzeitig hat vor allem die Einbindung der neuen europäischen Ökonomie in globale Reproduktionskreisläufe in Verbindung mit einer beschleunigten internen Marktintegration dazu beigetragen, dass über die europäische Ebene die wettbewerbsorientierte Modernisierung der nationalen Regulationssysteme aktiv gefördert wurde. Die europäische Währungs- und Finanzmarktintegration stützt sich auf die Strategie, die internationale Wettbewerbsfähigkeit der EU durch die Übernahme zentraler Elemente des US-amerikanischen Kapitalismusmodells (Schaffung transnational vernetzter und liberaler Wertpapiermärkte) sowie durch eine deflationäre Abwertungs- und Wettbewerbspolitik (Haushaltsdisziplin, restriktive Geld- und Lohnpolitik und Arbeitsmarktflexibilisierung) zu steigern (Grahl 2004; ders. 1997). Damit folgt die EU grundsätzlich nicht nur einem exportorientieren, mitunter neo-merkantilistischen Wachstumspfad und verbindet diesen mit einer voranschreitenden Finanzialisierung der Ökonomie, sondern ist auch durch eine zunehmende Selektivität zu Gunsten multinationaler (Export-) Unternehmen und der transnationalen Finanzindustrie geprägt.

Der Verlauf der Krise in Europa und der Charakter der europäischen Reaktionsweisen sind in diesem politökonomischen Kontext zu betrachten. Jedoch ist es für das Verständnis des europäischen Krisenmanagements ebenso von grundlegender Bedeutung, dass in Ergänzung zu einer politökonomischen Strukturanalyse auch die konkreten Initiativen, Wahrnehmungen und Koordinationsmechanismen der beteiligten Akteure in den Blick genommen werden. Dabei kann das derzeitige Krisenmanagement der Eurokrise nicht losgelöst von den Problemkonstellationen und Kompromissstrukturen der vorangegangenen Krisenphasen der Weltfinanzkrise beurteilt werden. Vielmehr sind die europäischen Reaktionsweisen vor allem durch eine wiederholte Verlagerung der Krisendynamik in Europa beeinflusst.

\section{Handlungsfelder und Entwicklung des europäischen Krisenmanagements}

Die Weltfinanzkrise von 2007 erfasste die europäische Ökonomie zum einen über die europäischen Banken und institutionellen Anleger, die ihr Kapital seit den 1990er Jahren im großen Umfang in den USA platziert hatten; und zum anderen, weil auch die europäischen Industrie- und Dienstleistungsunternehmen ihre Exportstrategien sehr stark auf den Weltmarkt ausgerichtet hatten und nun unter einem globalen Rückgang der Nachfrage zu leiden hatten (Becker 2011). Entsprechend dieser über die global vernetzten Finanzmärkte und Handelsbeziehungen 
Tabelle 1: Politische Handlungsfelder des europäischen Krisenmanagements

\begin{tabular}{|c|c|c|c|}
\hline & $\begin{array}{l}\text { Bankenrettungen und } \\
\text { Konjunkturprogramme }\end{array}$ & $\begin{array}{l}\text { Initiativen zur Finanz- } \\
\text { markt Re-Regulierung }\end{array}$ & $\begin{array}{l}\text { Euro Rettungsschirme } \\
\text { und Reform der EU } \\
\text { Strukturen }\end{array}$ \\
\hline $\begin{array}{l}\text { Krisen- } \\
\text { dynamik }\end{array}$ & $\begin{array}{l}\text { Banken- und } \\
\text { Wirtschaftskrise }\end{array}$ & $\begin{array}{l}\text { Finanzkrise und ihre } \\
\text { globale Ausbreitung }\end{array}$ & $\begin{array}{l}\text { Staatschuldenkrise } \\
\text { und Krise der } \\
\text { Währungsunion }\end{array}$ \\
\hline \multirow[t]{2}{*}{ Krisendiskurs } & Exogene Ursachen & Exogene Ursachen & Endogene Ursachen \\
\hline & $\begin{array}{l}\text { Fokus: europäische } \\
\text { Liquiditätskrise } \\
\text { und nationale } \\
\text { Ausnahmezustände }\end{array}$ & $\begin{array}{l}\text { Fokus: globales } \\
\text { Fehlverhalten von } \\
\text { Finanzakteuren und } \\
\text { Kontrolldefizite }\end{array}$ & $\begin{array}{l}\text { Fokus: nationale } \\
\text { Staatsschulden- und } \\
\text { Wettbewerbs- } \\
\text { problematik }\end{array}$ \\
\hline \multirow[t]{2}{*}{$\begin{array}{l}\text { Krisen- } \\
\text { management }\end{array}$} & $\begin{array}{l}\text { Strukturkonservatives } \\
\text { Krisenmanagement }\end{array}$ & $\begin{array}{l}\text { Partielle } \\
\text { Re-Regulierung der } \\
\text { Wertpapiermärkte }\end{array}$ & $\begin{array}{l}\text { Zurückhaltende } \\
\text { Rettungsschirme mit } \\
\text { Auflagen }\end{array}$ \\
\hline & $\begin{array}{l}\text { Neoliberale } \\
\text { Einbettung durch } \\
\text { Exit-Strategie der EU }\end{array}$ & $\begin{array}{l}\text { Fragmentierte } \\
\text { Bankenkontrolle }\end{array}$ & $\begin{array}{l}\text { Autoritäres } \\
\text { Austeritäts- und } \\
\text { Wettbewerbsregime }\end{array}$ \\
\hline
\end{tabular}

Quelle: Eigene Darstellung (für eine ausführliche Beschreibung siehe Bieling/Heinrich 2012)

vermittelten Krisenkanäle lässt sich nicht nur eine räumliche Differenzierung unterschiedlicher Krisendynamiken in Europa erkennen (Schmidt 2011), sondern der europäischen Krisenprozesse muss auch in unterschiedliche zeitliche Phasen unterteilt werden. Diesen Phasen liegen nicht nur jeweils unterschiedliche Krisendynamiken zu Grunde, sondern sie sind auch durch spezifische elitär-koordinierende diskursive Prozesse zur Etablierung einer (vermeintlich) organischen Kriseninterpretation und konkrete politische Entscheidungen und Reaktionsweisen des Krisenmanagements geprägt. Tabelle 1 gibt einen Überblick über die drei zentralen politischen Handlungsfelder des europäischen Krisenmanagements und die sich im Krisenverlauf verändernden Diskurse der Elitennetzwerke und Funktionsbedingungen der Reaktionsweisen wieder.

\section{Bankenrettungen und Konjunkturprogramme 2008/2009}

Die in Europa ergriffenen Maßnahmen zur Rettung systemisch relevanter Banken und zur Ankurbelung der Konjunktur reagierten vor allem auf die 2008 aufkeimende Banken- und Wirtschaftskrise in Europa. Das Übergreifen der Hypothekenkrise auf die weltweiten Derivatemärkte durch eine massive Entwertung von US-Kreditpapieren brachte nicht nur unzählige an diesen Geschäften beteiligte Banken in Europa in Refinanzierungsprobleme, sondern ließ auch 
die innereuropäischen Märkte für Interbankenkredite ins Stocken geraten. Die daraus resultierende Kreditklemme griff schnell auf die Realwirtschaft über. Marktkapitalisierte Unternehmen in Europa büßten rund 40\% ihres Marktwertes ein, Liquiditäts- und Investitionsengpässe und der krisenbedingte Rückgang der Exporte brachten die Konjunktur zum Erlahmen (Roth 2009: 25ff).

Der europäische Krisendiskurs in dieser Phase der Krise ist nicht nur von einer Betonung der Exogenität der Krisenursachen in den USA bestimmt (Fletcher 2010: 197). Von Seiten politischer und wirtschaftlicher Eliten in der EU wird die Krise vor allem als reine Liquiditätskrise und somit lediglich als ein kurzfristiger Störfaktor der wirtschaftlichen Entwicklung interpretiert und gleichsam in ein neoliberales Denkmuster überführt (COM 2008; ECB 2008). Bankenrettungen und Konjunkturprogramme werden als notwendige Ausnahmeerscheinung aufgrund der Dringlichkeit der Krise legitimiert und auf ihren möglichen wettbewerbsverzerrenden Charakter untersucht. Damit ist der Krise jeglicher systemischer Charakter genommen und das Leitbild eines schlanken Wettbewerbsstaates wird lediglich konzeptionell an den polit-ökonomischen Ausnahmezustand der Wirtschafts- und Bankenkrise angepasst, anstatt dass sich ein Paradigmenwechsel hin zu einem stärker keynesianisch orientierten Staatsverständnis entwickeln kann (Bieling 2010: 54f).

Entsprechend ist auch das nationale Krisenmanagement der europäischen Mitgliedstaaten 2008/2009 vor allem strukturkonservativ ausgerichtet und fokussiert sich maßgeblich auf die Rettung systemisch bedeutender Banken und vornehmlich angebotsorientierte Investitionsprogramme mit wenig progressiven Sozialinvestitionen, verteilungs- und/oder ökologiepolitische Gestaltungsaspekten (COM 2011; EZB 2010). Die EU verhält sich nach anfänglicher Passivität vornehmlich reaktiv zu den nationalen Reaktionsweisen und Lösungsansätzen. Die entsprechenden Kommissionrichtlinien gehen letztlich nicht über die bereits beschlossenen nationalen Maßnahmen hinaus und bilden vielmehr eine nachträgliche Absicherung, indem Bankenrettungen und Konjunkturprogramme ex-post mit den offiziellen EU Wettbewerbs- und Stabilitätsvorgaben in Einklang gebracht werden (Pisani-Ferry/Sapir 2010: 356f). Es findet vor allem eine neoliberale Einbettung der nationalen Krisenmanagementansätze und eine schnelle Rückkehr zu Ausgabendisziplin zur Sicherstellung der Wettbewerbsfähigkeit statt.

\section{Europäische Initiativen zur Re-Regulierung der Finanzmärkte 2009/2010}

Im Gegensatz dazu beziehen sich die europäischen Re-Regulierungsinitiativen von 2009/2010 direkt auf die Probleme, die sich aus der globalen Finanzkrise und deren globalen Ausbreitung ergeben und adressieren damit vor allem ein Defizit an Kontrolle von risikoreichen Wert- und Kreditpapiergeschäften. Durch die 
Verpackung von risikobehafteten Krediten und Hypotheken in undurchsichtige Derivate bildeten amerikanische Kreditpapiere nicht nur lukrative Anlagemöglichkeiten, sondern auch effektive Mittel zur Streuung von Risiken und erlebten am Vorabend der Krise eine massenhafte Verbreitung auf den globalen und europäischen Finanzmärkten (Mügge 2011.: 58f.). Das Platzen der US-Immobilienblase und ein damit einhergehender Anstieg der Prämien zur Absicherung von Kreditausfallrisiken löste somit schließlich eine globale Kettenreaktion aus, die über die globalen Derivatemärkte schnell auf die europäischen Banken übergriff und diese in erhebliche Refinanzierungsprobleme brachte.

Auch in dieser Phase der Krise wird von den europäischen Eliten vor allem die Exogenität der Krisenursachen hervorgehoben, wenngleich nun nicht mehr nur die USA als Sündenbock auftreten, sondern auch das individuelle und kollektive Fehlverhalten von Finanzakteuren auf den globalen Finanzmärkten thematisiert wird. Somit macht der im Februar 2009 veröffentlichte sog. Larosière Report, eines von der Kommission einberufenen Expertenkomitees, zwar weitreichende Vorschläge zur Überwachung des Finanzsystems auf europäischer und globaler Ebene, thematisierte die Krise jedoch auch weiterhin in einem gewohnt ideellen und vor allem stark technokratischen mikroökonomischen Denkmuster (Larosière et al 2009). Durch ein auf die Handlungen von Finanzakteuren, Ratingagenturen und Aufsichtsbehörden fokussiertes Kausalmodell mikroökonomisch oder gar massenpsychologisch begründeten Fehlverhaltens, werden die strukturellen Ursachen der Finanzkrise weitgehend ausgeklammert (Mayntz 2010: 4, 10). Dennoch sind hier einige neue, teilweise progressive diskursive Dynamiken zu erkennen, da nunmehr auch Defizite in der Regulierung der Finanzmärkte und risikobehafteter Wertpapiergeschäfte für die krisenhafte Entwicklung des globalen Finanzsystems mitverantwortlich gemacht werden (Wahl 2010).

Entsprechend weisen die 2009 von der Kommission verabschiedeten Richtlinien zur Re-Regulierung der europäischen Finanzmärkte partiell, aber vor allem in Bezug auf Wertpapiergeschäfte auch in die richtige Richtung. So werden im Zuge der G-20 Reformprozesse weitreichende Vorschläge zur Regulierung von Ratingagenturen, dem Verbot von OTC Derivaten und Leerverkäufen und der Beaufsichtigung von Investmentfunds erarbeitet (COM 2010; COM 2010a). Trotz dieser proaktiveren Strategie der EU sollten die Regulierungsbemühungen jedoch nicht überbewertet werden. Viele der unterbreiteten Initiativen wurden letztlich nicht, oder nur deutlich abgeschwächt umgesetzt. Und vor allem in Bezug auf die makroorientierte Kontrolle von Banken und institutionellen Anleger bleiben die Regulierungsbemühungen stark national fragmentiert und liegen teilweise weit hinter den Reformen zurück, die bereits vor der Krise in Bezug auf eine fortschreitende Integration der europäischen Finanzmärkte angedacht gewesen sind (Lannoo 2011: 5). 


\section{Euro-Rettungsschirme und Reform der EU Strukturen 2010-2012}

Schließlich reagieren die europäischen Rettungsschirme und auch die Reformen der europäischen Governance-Strukturen eindeutig auf eine sich mehr und mehr verschärfende Krise der europäischen Währungsunion. Im Zuge der Bankenkrise stieg der öffentliche Schuldenstand in der EU um ganze 24,3\%-Punkte an und vor allem die südeuropäischen Länder (Griechenland, Spanien, Portugal, Italien) und Irland gerieten 2010 über den Kreditkanal (stark zunehmende Liquiditätsengpässe europäischer Banken und steigende Zinsen auf den Kapitalmärkten) in massive Probleme ihre Staatschulden zu refinanzieren (Becker/Jäger 2011: 8f.). Auch wenn die betroffenen Staaten in Bezug auf die Höhe ihrer Verschuldung und deren grundlegenden Ursachen ganz erhebliche Unterschiede aufweisen ${ }^{2}$, so haben sie jedoch ein hohes strukturelles Handels- und/oder Leistungsbilanzdefizit gemein, das in Verbindung mit einer niedrigen Steuerquote zu einer starken Abhängigkeit von ausländischen Kapitalzuflüssen führt. Mit dem krisenbedingten Abzug dieses Kapitals gerät nunmehr die gesamte Währungszone an den Rand des Ruins, da aufgrund massiver makroökonomischer Ungleichgewichte innerhalb des europäischen Wirtschaftssystem die Defizitländer nicht nur abhängig sind von Kapitalzuflüssen aus den Überschussnationen, sondern die Herabstufung der Kreditwürdigkeit eines Mitgliedsstaates langfristig gesehen auch den Wert der gesamten europäischen Währung (und damit den internationalen Wettbewerbsvorteile für Exporte) und die Kreditwürdigkeit der anderen Mitgliedsstaaten der Währungszone bedroht (Altvater 2012: 276f.).

In dieser Phase der Krise thematisieren die europäischen Eliten vor allem die nationalen Staatschulden der Länder der europäischen Peripherie als wesentliche Ursache für die Krise des Euro (European Council 2010). Ganz im Gegenteil zu den vorherigen Krisendefinitionen sind die Interpretationen damit vor allem auf endogene Ursachen fokussiert. Die diskursive Koordinierung unterschiedlicher nationaler Präferenzen gelingt vor allem dadurch, dass sich die EU in den für die Krise relevanten Kompetenzbereichen auf einen langjährigen institutionalisierten Kompromiss der geld-, fiskal- und wettbewerbspolitischen Ausrichtung stützen kann. In Anknüpfung an die bereits im Lissabon-Vertrag getroffenen Vereinbarungen zur wirtschaftspolitischen Koordination werden haushaltpolitische Konsolidierung und eine verstärkte wirtschaftspolitische Koordination im Sinne einer Verschärfung des Stabilitäts- und Wachstumspaktes als entscheidende Maßnahmen zur Überwindung der makroökonomischen Ungleichgewichten in der EU angeführt (COM 2010b: 24f). D.h. die Ursachen der Krise werden

2 Für eine ausführlichere Darstellung der spezifischen nationalen Konstellationen siehe Becker/Jäger (2011) und Bischoff/Detje (2011). 
zwar in den makroökonomischen Ungleichgewichten der Währungsunion selbst gesucht, diese jedoch wiederum einseitig auf die Ausgaben- und Wettbewerbspolitik der Defizitländer zurückgeführt und somit nationale Fehlentwicklungen in der Wettbewerbsfähigkeit und Schuldenpolitik allein für die Ausweitung der Krise verantwortlich gemacht.

Entsprechend sind auch die Rettungspakete, die zur Unterstützung der Defizitländer geschürt werden, eher zurückhaltend und maßgeblich von nationalen Kalkülen geprägt, da sie nicht primär auf eine Reduzierung der Verschuldung und den Abbau der Ungleichgewichte, sondern vielmehr auf eine temporäre Stabilisierung der transnationalen Kreditbeziehungen und damit der Banken in der Eurozone abzielen (Bischoff/Detje 2011). Ähnlich wie in den anderen Handlungsfeldern sind die europäischen Institutionen auch hier zu Beginn der Krise überwiegend marginalisiert. Mit Einführung der Europäischen Finanzstabilisierungsfazilität (EFSF) und später des Europäischen Stabilitätsmechanismus (ESM) konnte jedoch ein Kompromiss zwischen der (vor allem deutschen) Ablehnung einer Transferunion und den (vor allem französischen) Ideen zu einer umfassenden EU Reform mit dem Ziel einer europäischen Wirtschaftsregierung gefunden werden (Young 2011: 12f.). Auf der einen Seite findet somit eine, wenn auch mit einer effektiven Kapazität von $500 \mathrm{Mrd}$. $€$ eher begrenzte Institutionalisierung von bilateralen Transferleistungen auf europäischer Ebene statt. Auf der anderen Seite aber werden die Kompetenzen, Ressourcen und vor allem die Überwachungsfunktionen der Europäischen Kommission (in Verbindung mit IWF und EZB) weitreichend reformiert und in ein nachhaltiges autoritäres, auf Austerität und Wettbewerbsdisziplin ausgerichtetes europäisches Finanzregime überführt. Mit Verabschiedung des sog. „Six-Packs“ und der Unterzeichnung des Euro-Plus-Pakts und des Fiskalpaktes in 2011 sind damit neue Instrumente des wirtschafts- und finanzpolitischen Managements auf europäischer Ebene institutionalisiert, die sich nicht nur auf autoritäre Disziplinierungsinstrumente und -maßnahmen stützen und bereits vorhandene supranationale Mechanismen der Austeritätspolitik verschärfen, sondern im Sinne einer restriktiven Wettbewerbslogik den Anpassungsdruck zur Überwindung der Krise allein auf die Länder mit einer defizitären Leistungsbilanz abladen (Katzer/Schlager 2011: 62f).

\section{Variegierter Krisenkonstitutionalismus: transnationale Dynamiken und Kompromissstrukturen}

Insgesamt lässt sich somit kaum ein progressiver, wenn überhaupt nur ein sehr moderater Wandel der grundlegenden Organisations- und Regulationsweise der europäischen Ökonomie im Zuge des Krisenmanagements erkennen. So weisen 
die Transformationsprozesse, z.B. durch die Initiativen zur Re-Regulierung der Finanzmärkte, teilweise zwar in die richtige Richtung, werden jedoch ebenso von repressiven Entwicklungen wie der Reform der europäischen Governance-Strukturen begleitet, so dass allenfalls von einer transformativen Reproduktion des europäischen Finanzmarktkapitalismus ausgegangen werden kann. Eröffneten sich während der Banken- und Wirtschaftskrise noch teilweise vielversprechende diskursive Fenster in Bezug auf die Rolle des Staates und eine Umorientierung weg von finanzmarktbasierten Wachstumpfaden, wurden diese schon früh durch die Etablierung einer Exit-Strategie in der EU hinweggefegt und in einen neoliberalen und wettbewerbsdisziplinären Interpretations- und Gestaltungsrahmen überführt. Stand die Finanzmarktpolitik zu Beginn der globalen Finanzkrise noch zunehmend im Fokus öffentlicher Kritik und wurden mögliche, weitreichende Modelle der Beteiligung der Banken an den Folgen der Krise debattiert, wird dieser Diskurs spätestens mit der Eurokrise durch eine Kritik des Schuldenmachens und überhöhter Löhne einiger weniger Länder in der EU abgelöst. Eine produktive und progressive Konjunkturpolitik war und ist damit nicht auf der europäischen Agenda zu finden, vielmehr wird der Weg zurück zu einer austeritätspolitischen Wettbewerbsagenda geebnet, die über einen transnationalen Finanzmarktkapitalismus hinausweisende Transformationsperspektiven schon früh erfolgreich unterbindet.

Ebenso haben sich die sozialen und machtpolitischen Netzwerke in der EU, wenn überhaupt, nur sehr punktuell verschoben. Ein von transnationalen neo-merkantilistischen und finanzkapitalistischen Interessen dominierter Machtblock in der EU, der neben den dominanten europäischen extrovertierten Produktivkapitalien und Finanzkapitalgruppen (die stark in die transnationalen und globalen Produktions- und Reproduktionskreisläufe eingebunden sind) die (Gläubiger-) Nationen umfasst, die vornehmlich auf Exporte und eine elitäre Finanzialisierung ausgerichtet sind, hat weitestgehend Bestand (Becker/Jäger 2011). Sich mitunter ergebende Konflikte, die an solchen Stellen auftreten, an denen die unterschiedlichen Akkumulationsstrategien in Widerspruch zueinander geraten, können bisher durch einen neoliberalen Basiskonsens zusammen gehalten werden. Dieser etablierte europäische Machtblock und vor allem die an ihm beteiligten Kapitalgruppen profitieren von einer Politik, die auf die Rettung von an risikoreichen globalen Geschäften beteiligten Banken ausgerichtet ist und angebotsorientierten Konjunkturprogrammen zur Wiederbelebung der nationalen Schlüsselindustrien in der ersten Phase der Krise. Gleichzeitig sind die Re-Regulierungsinitiativen der Finanzmärkte in der EU nur graduell und teilweise allein symbolischen Charakters und können damit die Anlagestrategien der europäischen Finanzakteure weder entscheidend einschränken noch stellen sie eine klare Abkehr einer auf die Finanzmärkte ausgerichteten Politik dar (Trost 
2011). Auch wenn vor allem institutionelle Anleger und Rating Agenturen durch die von der Kommission angedachten und durchgeführten Reformen in der EU sicherlich nicht zusätzlich an Macht gewinnen, so sind die Richtlinien aber auch nicht in der Lage, das Wiedererstarken dieser Fraktionen des Finanzkapitals nach der globalen Finanzkrise entscheidend aufzuhalten (Ayadi et al. 2011:39f). Und auch von der Rückkehr zu einer disziplinären Austeritäts- und Wettbewerbspolitik sowie stärkeren Überwachungsmechanismen in der EU profitieren vor allem die Exportnationen und Gläubigerstaaten Europas, während die Defizitländer der europäischen Peripherie zusätzlich an Gestaltungsspielraum und Perspektive verlieren. Die europäischen Rettungsschirme und Stützungsmaßnahmen dienen dabei nicht nur den großen europäischen Banken, die ihre Gelder in diesen Ländern platziert haben, sondern die Verschärfung der europäischen Austeritätsund Deflationspolitik sichert ebenso die internationalen Wettbewerbsvorteile europäischer multinationaler Exportunternehmen auf dem Weltmarkt.

Damit ist das europäische Krisenmanagement ganz wesentlich durch eine doppelte Einbettung der EU Politik charakterisiert: auf der einen Seite werden die europäischen Reaktionen auf die Krise durch die globale Integration in das Dollar Wall Street Regime (DWSR) von außen stimuliert (Gowan 1999), auf der anderen Seite blieben die Managementstrategien aber auch ganz entscheidend in spezifischen nationalen Finanz- und Kapitalismusmodellen verhaftet, die eine signifikante Varianz rechtlicher, institutioneller und operativer sowie kultureller nationaler Besonderheiten und damit auch Reaktionsweisen generieren. Diese doppelte Einbindung im Sinne eines 'variegierten' Kapitalismus (Jessop 2012) erklärt nicht nur den europäischen Verlauf der Weltfinanzkrise in Europa, sondern führt besonders in Bezug auf die europäischen Reaktionsweisen zu einer teilweise widersprüchlichen sicherlich aber konfliktgeladenen Kompromissstruktur, die ganz maßgeblich durch die Gestaltungsmacht der unterschiedlichen nationalen und europäischen staatlichen und zivilgesellschaftlichen Akteure zum Ausdruck kommt. Die Krisenreaktionen in der EU sind sowohl durch die transatlantischen Wirtschaftsbeziehungen und die Einbindung in den US-amerikanischen Reproduktionskreislauf, als auch durch heterogene nationale Konstellationen, Diskurse und Strategien der Krisenbewältigung geprägt.

In Bezug auf die Bankenrettungs- und Konjunkturmaßnahmen bilden die entsprechenden Kommissionrichtlinien nicht viel mehr als eine nachträgliche Absicherung der stark heterogenen nationalen Maßnahmen, die zwar einerseits weit genug ausgelegt sind, um die Pragmatismen aller Mitgliedstaaten unter einem Schirm zu vereinen, die aber andererseits vor allem auf Drängen der in globale Produktions- und Reproduktionsketten eingebundenen und im Zuge expansiver Ausgabenprogramme um die eigene Wettbewerbsfähigkeit bangenden europäischen Handels- und Industrieunternehmen in eine globale Wettbewerbslogik und 
die Aufrechterhaltung freier Handelsbeziehungen überführt, bzw. zurückgeführt werden. Die Initiativen zur Re-Regulierung der Finanzmärkte sind hingegen von stärkeren Konflikten geprägt, in denen vor allem die in die globalen Reproduktionskreisläufe eingebetteten europäischen und nationalen Finanzkapitalgruppen und nationale Notwendigkeiten der Regulierung, ebenso aufeinandertreffen wie unterschiedliche nationale Finanzmodelle, die entweder auf eine überwiegend neomerkantilistische Akkumulationsweise, oder aber einer primär finanzgetriebenen Akkumulation beruhen. Hier bilden die Initiativen der Kommission vom Anfang eine Kompromisslösung zwischen einem eher angelsächsischen „market-taking“ orientierten und einem stärker rheinischen „market-making“ orientierten Ansatz (Quaglia 2010). Und während die Kommission vor allem den Ausbau der europäischen Machtposition in den internationalen Währungs- und Finanzbeziehungen im Auge hat, sind es die in globale Reproduktionskreisläufe des DWSR integrierten europäischen Finanzkapitalgruppen die, mit Unterstützung jener Nationalstaaten mit einem primär elitären Finanzialisierungsmodell (bspw. Großbritannien), diese Reformen blockieren bzw. nachträglich verwässern. Und auch in Bezug auf die Eurokrise sind die unmittelbaren Reaktionen auf die Staatschuldenkrise vornehmlich durch Kompromisse zwischen Gläubiger- und Defizitländern geprägt und von nationalen Kalkülen und transanationalen Konflikten in Bezug auf ein einheitliches europäisches Finanzmodell, aber auch auf die Rolle und Zukunft des europäischen Integrationsprozesses im Allgemeinen durchzogen. Denn durch die stark ins Wanken geratene Währungsunion sind nunmehr die Grenzen des bisherigen (Krisen-)Managements und Gestaltungsrahmen der EU erreicht und eine erhebliche Ausweitung der Kompetenzen und finanziellen Ressourcen der Institutionen von Nöten, um einen Zusammenbruch des neoliberalen EU-Systems zu verhindern (Deppe 2011). Dies verschärft die nationalen Disparitäten in Bezug auf die Rolle und Funktion der EU, wobei sich hier vor allem intergouvernementale Formen einer überwiegend negativen Wirtschaftsintegration und stärker supranationale Formen einer politischen Vergemeinschaftung gegenüber stehen.

Bisher können all diese Positionen noch durch eine Strategie der Vertiefung bestehender Disziplinierungsmaßnahmen zur Aufrechterhaltung der derzeitigen Handels-, Kredit- und Machtverhältnisse innerhalb der EU zusammengehalten werden. Davon profitieren nicht nur die exportorientierten Überschussländer, die die Etablierung einer langfristigen Transferunion verhindern wollen und die Länder deren Banken als transnationale Gläubiger der Defizitländer auftreten und die derzeitigen Verschuldungsstrukturen zu verteidigen zu versuchen (Bischoff/Detje 2011). Es sind auch die in globale Produktionsketten eingebundenen europäischen Exportunternehmen, deren Akkumulationsstrategien sich mit einer auf Wettbewerbsdisziplin und deflationäre Abwertung ausgerichteten EU- 
Politik decken, sowie die global orientierte europäische Finanzindustrie, deren Investitions- und Anlagestrategien durch die austeritätspolitische Ausrichtung und Eingliederung der EU in die Funktionsweise des DWSR begünstigt werden, die von dem Krisenmanagement maßgeblich profitieren.

\section{Die Desartikulation alternativer Ansätze: Diskursive Dynamiken und Kampffelder}

Gleichzeitig werden alternative Entwicklungen jeglicher Form und eine Neujustierung der politischen Prioritäten in der EU vor allem durch eine elitäre diskursive und organisationspolitische Desartikulation sowie die ständige Verschiebung der politischen Kampfelder im Keim erstickt. Es fällt auf, dass sich die Aufmerksamkeit der politischen Entscheidungsträger, ebenso wie die der breiten Öffentlichkeit im Zuge sich kontinuierlich verändernden Krisendynamiken und unterschiedlicher Krisenproblematiken, ebenso schnell verschieben, wie die politischen Kämpfe, die mit dem Krisenmanagement dieser Probleme einhergehen.

Somit spiegeln die Krisenreaktionen in Bezug auf die Re-Regulierung der europäischen Wertpapiermärkte und risikoreicher Anlagegeschäfte zwar einen neuen Kompromiss in Bezug auf die Anerkennung von Regulierungsdefiziten in einigen Bereichen des globalen Finanzsystem wider, betten diese aber gleichermaßen in gewohnt neoliberale Geschäftspraktiken und Denkmuster ein. Dies lässt sich unter anderem daran erkennen, dass es gelingt, die verteilungspolitischen Debatten in Bezug auf eine Beteiligung des Bankensektors an der Finanzkrise zwar mit einer Diskussion um eine Finanztransaktionsteuer auch auf europäischer Ebene aufzugreifen, dieser Diskurs aber gleichzeitig erheblich an Brisanz verliert, indem er fast vollkommen losgelöst von den massiven Bankenrettungsmaßnahmen der vorherigen Krisenphase geführt wird. Die Diskussionen konzentrieren sich vor allem auf das Mikro-Fehlverhalten von Finanzakteuren auf den globalen Wertpapiermärkten, was zu einer Technokratisierung des Diskurses beiträgt. Ebenso liegt dem Prozess der Re-Regulierungsmaßnahmen eine viel größere Anlaufzeit zu Grunde, als den ad-hoc getroffenen Entscheidungen zur Überwindung der Banken- und Wirtschaftskrise. Dies erlaubt den 2008 noch stark in der Kritik stehenden und materiell angeschlagenen Banken und institutionellen Anlegern sich neu zu formieren und ihren Einfluss auf das Krisenmanagement 2009 wieder weitaus selbstbewusster anzugehen, was sich nicht nur in einer Ausweitung der Lobbyanstrengungen der Finanzindustrie ab 2009 wiedererkennen lässt (CEO 2011). Dieser Zusammenhang gilt auch für den Einfluss neoliberal ausgerichteter europäischer Expertenkomitees, wie der Larosière Gruppe, die 2007/2008 allein schon aufgrund der Dringlichkeit der Krisenreaktionen weitaus weniger gefragt 
waren, und die sich in dieser Phase von der allgemeinen Orientierungslosigkeit etablierter Mainstream-Ansätze zur Erklärung der Krise erholen musste. Mit dem zunehmenden Verlauf der Krise geht ein Wiedererstarken von in der Hochphase der Krise stark verunsicherten Ökonomen, Spezialisten und Think Tanks einher, die nunmehr zu ihren alten Denkmustern zurückfinden und diese wieder offen vertreten.

Das Wiedererstarken etablierter neoliberaler Denkmuster und etablierter finanzmarktkapitalistischer Paradigmen gilt ebenso für das Handlungsfeld der Eurorettung. Dort wird der elitäre Diskurs ebenfalls losgelöst von den Folgekosten der globalen Banken- und Finanzkrise geführt. Eine Verbindung der Krise zu den Problematiken vorheriger Krisenkonstellationen, wie der Rolle von Rating Agenturen, ungezügelten Kapitalmärkten oder innovativer Finanzinstrumente konnte überwiegend verhindert werden. Dies lässt sich unter anderem daran erkennen, dass die Eurokrise im Kontrast zu den anderen Handlungsfeldern vor allem als endogenes Phänomen analysiert und bearbeitet wird, was z.B. eine Verbindung zu den kostenintensiven Bankenrettungsmaßnahmen kategorisch erschwert. Gleichzeitig impliziert dieser endogene Fokus, dass die Instrumente zur Überwindung der Krise durch eine Reform des eigenen supranationalen Rahmens herbeigeführt werden können (Schelkle 2011). Wurde in der Banken-, Wirtschafts- und Finanzkrise noch versucht den europäischen Kompetenzbereich der EU Institutionen von den Reformen auszuschließen, nicht zuletzt weil dies wahrscheinlich zu einer stärkeren Nationalisierung oder einer Neujustierung der Ziele hätte führen müssen, bietet die Eurokrise für die politischen und wirtschaftlichen Eliten nun die passende Gelegenheit die bereits etablierten Disziplinierungsmechanismen weiter zu verschärfen und den (Überwachungs-) Gestaltungsrahmen der EU auszubauen.

Ebenso zeigt sich im europäischen Krisenmanagement, dass in allen Phasen der Krise eine erhebliche Engführung im europäisch koordinierenden Diskurs stattfindet, die nicht selten von den europäischen Eliten, Interessengruppen und Expertengremien gefördert wird und die letztlich dazu beiträgt, etwaige Diskursverschiebungen (wie z.B. in Bezug auf die Rolle des Staates) in ein neoliberales und wettbewerbsorientiertes Denkmuster zu überführen (Ljungman 2011). In Bezug auf die Bankenrettungen und Konjunkturprogramme bestimmt ein auf die geldpolitischen, bankenorientierten oder wachstumspolitischen exogenen Fehler in den USA und auf Liquiditätsengpässe systemisch relevanter Banken ausgerichteter Diskurs die einschlägigen Krisendiagnosen. Durch eine Verteidigung der Liquiditätskrise als Ausnahmezustand und eine Einbettung der Diskussion in etablierte neoliberale Muster eines schlanken Wettbewerbsstaates werden sowohl verteilungspolitische als auch staatskonzeptionelle Fragen im Kern erstickt. Dieser Diskurs ist insofern verkürzt, als dass einseitig auf die Rettung systemisch bedeutender Banken 
und angebotsorientierter Investitionsprogramme ausgerichtete Krisendiagnosen die Krise nicht als global und europäisch vermitteltes Problem einer anhaltenden Kaufkraftlücke (Unterkonsumption) und Profitklemme anlagesuchender Finanzakteure als Folge einer Überakkumulationskrise verstehen (Unger 2010).

In Bezug auf die Re-Regulierungsinitiativen der Finanzmärkte findet eine diskursive Engführung vor allem durch einen expliziten Fokus auf das auf der Mikro-Ebene angesiedelte Verhalten einzelner bestimmter Finanzmarktakteure und Finanzmarktgeschäfte auf den globalen (exogenen) Finanzmärkten statt. Anstatt die systemischen Prozesse, durch die ein dereguliertes globales Finanzsystem aus sich selbst heraus zu Instabilität tendiert, zu thematisieren (Minsky 1982), findet in einem stark auf technokratische Prozesse und Handlungen von Finanzakteuren, Rating Agenturen und Aufsichtsbehörden fokussierten Kausalmodell im europäischen Diskurs keine umfassende Systemkritik statt. Obwohl die Anerkennung der Tatsache, dass Defizite in den Regulierungsmechanismen globaler Finanzmärkte einen Teil des Problems darstellen, bereits eine gewisse Diskursverschiebung impliziert, findet kein grundsätzlicher Paradigmenwechsel in Bezug auf die strukturell destabilisierende Wirkung deregulierter globaler Finanzmärkte statt.

Und auch in Bezug auf die Eurokrise sind maßgebliche Verkürzungen im europäischen Diskurs zu erkennen, indem nunmehr allein die endogenen makroökonomischen Ungleichgewichte der Eurozone als Krisenursache diagnostiziert werden, welche wiederum auf eine mangelnde Wettbewerbsfähigkeit und das unverbesserliche Schuldenmachen einzelner Mitgliedstaaten zurückgeführt werden. Der europäische Diskurs konzentriert sich damit vor allem auf die Rolle der staatlichen Schuldner in der Krise, während dieses Problem allein in seiner nationalen und monetären Dimension erfasst wird, anstatt die Problematik privater Schulden (wie im Fall Spaniens, Irlands oder Portugals), die Überführung privater in öffentliche Schulden im Zuge der Banken und Finanzkrise, die transnationale Verflechtung der europäischen Kreditbeziehungen (und damit die Rolle der Banken in den Gläubigernationen) und die Ungleichgewichte in der Entwicklung des europäischen Handelsverkehrs, die aus einer mangelnden Binnennachfrage und den restriktiven Lohnregimen in den Exportnationen (und damit aus einer asymmetrischen Integration) resultieren, als Krisenursache mit einzubeziehen (Bieling 2011).

\section{Ausblick: die Krise des Krisenmanagements}

Auch wenn das europäische Krisenmanagement zu einer zeitweiligen Stabilisierung des Finanzsektors und der transnationalen Verschuldungs- und 
Handelsbeziehungen in Europa beitragen kann, so werden die grundlegenden Strukturprobleme der europäischen Ökonomie jedoch nicht entscheidend bearbeitet. Während die Bankrettungen zwar den Zusammenbruch der globalen Finanzmärkte verhindern konnten, erholten sich die Preise für Finanzaktiva ab 2009 wieder und sorgten damit auch für eine Revitalisierung der Anlagestrategien profitsuchender Finanzakteure (Becker 2011: 25). Eine bereinigende Wirkung der Überakkumulation durch eine Entwertung des Kapitals bleibt damit aus, wodurch sich die Kreisläufe des spekulativen und produktiven Kapitals heute nunmehr erneut auseinander entwickeln und den Aufbau einer neuen Spekulationsblase anfeuern, die letztlich eine weitere Krisenphase und wirtschaftliche Instabilitäten hervorbringen wird. Überwiegend symbolische, oder im politischen Prozess verwässerte Versuche einer Re-Regulierung der europäischen Wertpapiermärkte wirken diesem Trend nicht entgegen und lassen zahlreiche Problematiken sowie nationale Lücken unberücksichtigt (Wahl 2010: 35). Gleichzeitig sind die Stützungsmechanismen in der Eurokrise nicht nur mit erneut steigenden Kosten verbunden, sie sorgen auch für eine Vertiefung der Spaltungslinien zwischen Zentrum und Peripherie. Bisher sind weitreichende Lösungen zur Überwindung der strukturellen Ungleichgewichte in der EU durch die marktliberalen Machtnetzwerke aus Finanzkapital, Großindustrie und konservativen Regierungen verhindert worden. Stattdessen soll ein autoritäres Austeritätsregime zu einer Verbesserung der Finanzierungsmöglichkeiten beitragen, letztlich aber wird dadurch nur eine langanhaltende Rezession in den betroffenen Ländern eingeleitet, die nicht zu einer nachhaltigen Senkung der Verschuldung führt, sondern die Wahrscheinlichkeit einer wettbewerbspolitischen Deflationsspirale in der gesamten EU erheblich vergrößert (Altvater 2012: 284f.).

Das europäische Krisenmanagement setzt damit nicht nur neue Krisendynamiken frei, sondern sorgt auch dafür, dass die Widersprüche und Konflikte des europäischen Integrationsprozesses fortbestehen und sich mitunter entscheidend verschärfen. Dies ergibt sich nicht zuletzt daraus, dass, abgesehen von der Geldund Fiskalpolitik, weite Teile der finanz- und makroökonomischen Regulation auf europäischer Ebene fragmentiert bleiben. Die Überwindung der asymmetrischen supranationalen Integration birgt jedoch gerade in Bezug auf eine mögliche Fortschreitung der europäischen Finanzmarktintegration entscheidende Konfliktpotentiale zwischen primär finanzialisierten Ökonomie, wie Großbritannien, und produktiv exportorientierten Volkswirtschaften, wie Deutschland, in sich.

Gleichzeitig ist das europäische Krisenmanagement vor allem allein auf die Interessen der führenden Fraktionen des Herrschaftsgefüges - des transnationalen Finanz- und Industriekapitals - ausgelegt. Damit erschwert die Krise langfristig gesehen nicht nur die Organisation des politischen Interesses innerhalb des europäischen Blocks an der Macht, sondern auch die Einbindung subalterner 
Kräfte in den Selbigen (Oberndorfer 2012: 57). Bisher werden die europäischen Kompromissstrukturen noch durch einen starken marktliberalen Konsens und die Pfadabhängigkeit einseitig auf wettbewerbssteigernde und austeritätspolitische Zielsetzungen fokussierte europäische Institutionen zusammengehalten (MaCartney 2011: 157). Jedoch sind weitere Integrationsschritte und Veränderungen der institutionellen europäischen Strukturen nötig, um die Union in ihrer derzeitigen Konfiguration aufrecht zu erhalten, welche erhebliche Konflikte mit sich bringen kann. Nicht zuletzt deshalb, weil es gerade diese marktliberalen und autoritären Reformen in Reaktionen auf die Krise sind, die den Klassencharakter der europäischen Politik immer weiter zu Tage treten lassen.

Die Kosten der Krise wurden bisher vor allem auf weite Teile der Bevölkerung in der europäischen Peripherie, lohnabhängig und prekär beschäftigte ArbeitnehmerInnen und den EmpfängernInnen von Sozialleistungen abgeladen. Mit dieser Politik wächst die soziale und politische Kritik nicht nur an der Ausrichtung der nationalen und europäischen Maßnahmen, sondern auch an den herrschenden Verhältnissen im Allgemeinen. Speziell in den Ländern Südeuropas stellen Generalstreikbewegungen und Massendemonstrationen die Wirksamkeit und Selektivität nationaler Sparprogramme offen in Frage. Gleichzeitig attackieren soziale Proteste die herrschenden Ungleichheiten und verteilungspolitischen Ungerechtigkeiten, die sich durch die Krise einmal mehr offenbart haben (Deppe 2011: 25). Diese Proteste verweisen auf eine sich verschärfende Krise nationaler und europäischer Legitimation. Bereits seit dem Wandel der europäischen Integrationsweise in den 1980er Jahren befindet sich die EU nunmehr in einer Jahrzehnte lang andauernden „Post-Maastricht-Krise“, die nicht nur durch die Erosion eines auf ökonomischen Erfolg beruhenden permissiven Konsens, sondern auch durch eine zunehmende Krise politischer Führung gekennzeichnet ist (Beckmann et al 2006).

Die „Output Legitimität“ des europäischen Projekts wird durch die Krise weiter geschwächt, schon allein da der europäische Integrationsprozesse nunmehr nicht nur keine wesentlichen Lösungen für die anhaltenden Probleme wirtschaftlicher Entwicklung bietet, sondern vielmehr als ein maßgebliches Problem von krisenhaften Prozessen in Erscheinung tritt. Es fehlt der EU an einer direkten Legitimationsbeziehung zur europäischen Bevölkerung (Urban 2011: 45f.). Ebenso offenbart das europäische Krisenmanagement eine ernstzunehmende Krise repräsentativer Demokratieformen. Auf der einen Seite lassen vornehmlich auf Regierungsvereinbarungen gestützte europäische Reformen eine Teilnahme des Europäischen Parlaments vermissen und beruhen allein auf den Vorschlägen von überwiegend männlichen technokratischen Bürokratenzirkeln und intransparenten und elitären Entscheidungsprozesse ohne jegliche Beteiligung der europäischen BürgerInnen (ebd.: 43f.). Auf der anderen Seite aber sind auch 
die Konsequenzen der beschlossenen Maßnahmen nur als demokratiepolitischer Sündenfall zu bewerten, da nunmehr entscheidende Kompetenzen und (de facto) rechtliche Befugnisse in Bezug auf Kernbereiche wirtschaftspolitischer Weichenstellungen der Nationalstaaten auf BürokratInnen der Europäischen Kommission übertragen werden und sich damit jeglicher demokratischer Kontrolle entziehen (Klatzer/Schlager 2011: 67f.).

Das autoritäre Austeritäts- und Wettbewerbsregime in Europa weist damit in keine erstrebenswerte Zukunft. Durch die kurzfristigen Stabilisierungen der europäischen Banken- und Finanzsektoren werden die Voraussetzungen für eine erneute Finanzkrise geschaffen. Gleichzeitig wälzt ein auf Demokratieverzicht basiertes Überwachungs- und Durchsetzungsmodell fiskalischer Konsolidierung und wettbewerbspolitischer Disziplinierung in Europa die Kosten der Krise allein auf die abhängig arbeitende oder arbeitssuchende Bevölkerung ab und verstetigt damit etablierte Verschuldungs- und neoliberale Machtbeziehungen. Nicht zuletzt deshalb ist die Ausweitung des Drucks sozialer und gewerkschaftlicher Bewegungen, die sowohl die transnationalen Macht- und Kräfteverhältnisse thematisieren, als auch die diskursiven Deutungskämpfe annehmen, heute zwingend erforderlich - auch, oder gerade weil die Möglichkeit einer emanzipatorische Transformation der Europäischen Union derzeit in weiter Ferne zu liegen scheint.

\section{Literatur}

Altvater, Elmar (2012): From Subprime Farce to Greek Tragedy: The crisis dynamics of financial driven capitalism. In: Panitch, Leo/Albo, Greg/Chibber, Vivek (Hg.): The Crisis and the left. Socialist Register 2012, Vol. 48.

Ayadi, Rym/Arbak, Emrah/De Groen, Willem Pieter (2011): Business Models in European Banking: a pre-and post-crisis screening. Center for European Policy Studies.

Becker, Joachim (2011): EU: von der Wirtschafts- zur Regulationskrise. In: Z-Zeitschrift für Marxismus 85, 10-29.

Becker, Joachim/Jäger, Johannes (2011): European Integration in Crisis. Paper presented at the $17^{\text {th }}$ EuroMemo Workshop on Alternative Ecconomic Policy. Vienna, 16.-18.9.2011

Beckmann, Martin/Deppe, Frank/Heinrich, Mathis (2006): In schlechter Verfassung? Ursachen und Konsequenzen der EU-Verfassungskrise. In: PROKLA 36 (3), 307-324.

Bieling, Hans-Jürgen (2011): Vom Krisenmanagement zur neuen Konsolidierungsagenda der EU. In: Prokla 41 (2), 173-194.

- (2010): Metamorphosen des “integralen Staates”. Konkurrierende Leitbilder in der Krisendiskussion. In: Altvater, Elmar et al. (Hg.): Die Rückkehr des Staates nach der Finanzkrise. Hamburg: VSA-Verlag, 37-60.

Bieling, Hans-Jürgen/Heinrich, Mathis (2012): Die Transformation des europäischen Finanzsystems. Krisendynamiken, Initiativen und Konflikte. Berlin: Reihe „Analysen“ der RosaLuxemburg-Stiftung, i.E.

Bischoff, Joachim/Detje, Richard (2011): Die Große Krise der Euro-Zone. Konstruktion - Fehlsteuerung - Alternativen In: Bischoff, Joachim et al (Hg): Europa im Schlepptau der Finanzmärkte. Hamburg: VSA Verlag, 65-127. 
Corporate Europe Observatory (CEO) (2011): Business against Europe. Business Europe celebrates social onslaught in Europe. http://www.corporateeurope.org/publications/ business-against-europe-businesseurope-celebrates-social-onslaught-europe

Deppe, Frank (2011): Der Weg in die Sackgasse. Eine Kurzgeschichte der europäischen Integration. In: Bischoff, Joachim et al (Hg): Europa im Schlepptau der Finanzmärkte. Hamburg: VSA Verlag, 9-29.

European Central Bank (ECB) (2010): Euro Area Fiscal Policies and the Crisis. Occasional Paper Series No. 109. April 2010. Frankfurt/Main.

- (2008): Recommendations of the Governing Council of the European Central Bank on the pricing of recapitalisations. Frankfurt/Main.

European Commission (COM) (2011): Report from the Commission: State Aid Scoreboard. Report on state aid granted by the EU Member States. Autumn 2011, Update. COM(2011) 848 final.

- (2010): Proposal for a Directive of the European Parliament and of the Council on OTC derivatives, central counterparties and trade repositories. $\operatorname{COM}(2010) 484 / 5$ final.

- (2010a): Proposal for a Directive of the European Parliament and of the Council on Short Selling and certain aspects of Credit Default Swaps. $\operatorname{COM}(2010) 482$ final.

- (2010b): EUROPE 2020. A European strategy for smart, sustainable and inclusive growth. Brussels. $\operatorname{COM}(2010) 2020$.

- (2008): Communication from the Commission: The recapitalisation of financial institutions in the current financial crisis. $\operatorname{COM}(2008) 8259$ final.

European Council (2010): Strengthening Economic Governance in the EU. Final Report of the Task Force to the European Council. Brussels.

Fletcher, Tully (2009): The European Union: from impotence to opportunity? In: t'Hart, P./ Kindall, K. (Hg.): Framing the global economic downturn. Crisis rhetoric and the politics of recession. Australien National Unveristy.

Grahl, John (2004): The European Union and American Power, in: Panitch, Leo/Leys, Colin (Hg.): The Empire Reloaded. Socialist Register 2005, London: Merlin Press, 284-300.

- (1997): After Maastricht. A guide to European monetary union. London: Lawrence and Wishart Limited.

Gowan, Peter (1999): The Global Gamble. Washington's Faustian Bid for World Dominance. London; New York: Verso.

Harvey, David (2010): The enigma of capital: and the crises of capitalism. New York: Oxford University Press.

Jessop, Bob (2012): Spielarten des Kapitalismus und variegierter Kapitalismus. In: Bruff, Ian et al. (Hg.): Kapitalismus, Kontinuität, Krise. Münster: Westfälisches Dampfboot. i.E.

Klatzer, Elisabeth/Schlager, Christa (2011): Europäische Wirtschaftsregierung - eine stille neoliberale Revolution. In: Kurswechsel 29(1), 61-81.

Lannoo, Karel (2011): The EU's Response to the Financial Crisis: A mid-term review. CEPS Policy Brief, No. 241, April.

Larosière, Jacques de et al (2009): Report from the High-Level Group on Supervision in the EU. Brussels.

Ljungman, Pia (2011): Constructing the European Sovereign Debt Crisis, hegemonising austerity. A narrative of European neoliberal governmentality. MA Dissertation.

MaCartney, Huw (2011) Variegated Neoliberalism: EU varieties of capitalism and international political economy. Routledge.

Mayntz, Renate (2010): Die transnationale Ordnung globalisierter Finanzmärkte. Was lehrt uns die Krise? MPIfG Working Paper 10/8.

Minsky, Hyman P. (1982): Can "It" Happen Again? Essays on Instability and Finance, Armonk/ New York: M.E. Sharpe. 
Mügge, Daniel (2011): Kreditderivate als Ursachen der globalen Finanzkrise: Systemfehler oder unglücklicher Zufall? In: Kessler, Oliver (Hg.): Die Internationale Politische Ökonomie der Weltfinanzkrise. Wiesbaden: VS Verlag, 53-74.

Oberndorfer, Lukas (2012): Hegemoniekrise in Europa. Auf dem Weg zu einem autoritären Wettbewerbsetatismus? In: Forschungsgruppe 'Staatsprojekt Europa' (Hg.): Die EU in der Krise. Zwischen autoritärem Etatismus und europäischem Frühling. Westfälisches Dampfboot, 50-72.

Pisani-Ferry, Jean and Andrè Sapir (2010): Banking crisis management in the EU: an early assessment. In: Economic Policy, April 2010, 341-373.

Quaglia, Lucia (2010): The "Old" and "New" Politics of Financial Services Regulation in the EU. OSE Paper No.2.

Roth, Karl Heinz (2009): Die Globale Krise. Globale Krise - globale Proletarisierung - Gegenperspektiven. Band 1. Hamburg: VSA-Verlag.

Trost, Axel (2011): Das Kasino bleibt geöffnet. Die Regulierung der Finanzmärkte und was daraus geworden ist. In: Blätter für deutsche und internationale Politik 2/2011, 75-84.

Schelkle, Waltraud (2011): A tale of two crises: the euro area in 2008/09 and in 2010. In: European Political Science: 10, 375-383.

Schmidt, Ingo (2011): European Capitalism: Varieties of Crisis. In: Alternate Routes - A Journal of Critical Research 22, 71-86.

Young, Brigitte (2011): Economic Governance in the Eurozone: A New Dawn? In: Economic Sociology 12(2), 11-16.

Wahl, Peter (2010): Fighting Fire with Buckets A Guide to European Regulation of Financial Markets. WEED - World Economics, Environment \& Development Assoc.

Unger, Brigitte (2010): Wer ist schuld an der Finanzkrise. In: Kurswechsel 25(1), 24-37.

Urban, Hans-Jürgen (2011): Das neue Europa: stabil und autoritär? Europas Weg in einen neuen Autoritarismus. In: Bischoff, Joachim et al (Hg): Europa im Schlepptau der Finanzmärkte. Hamburg: VSA Verlag, 30-64.

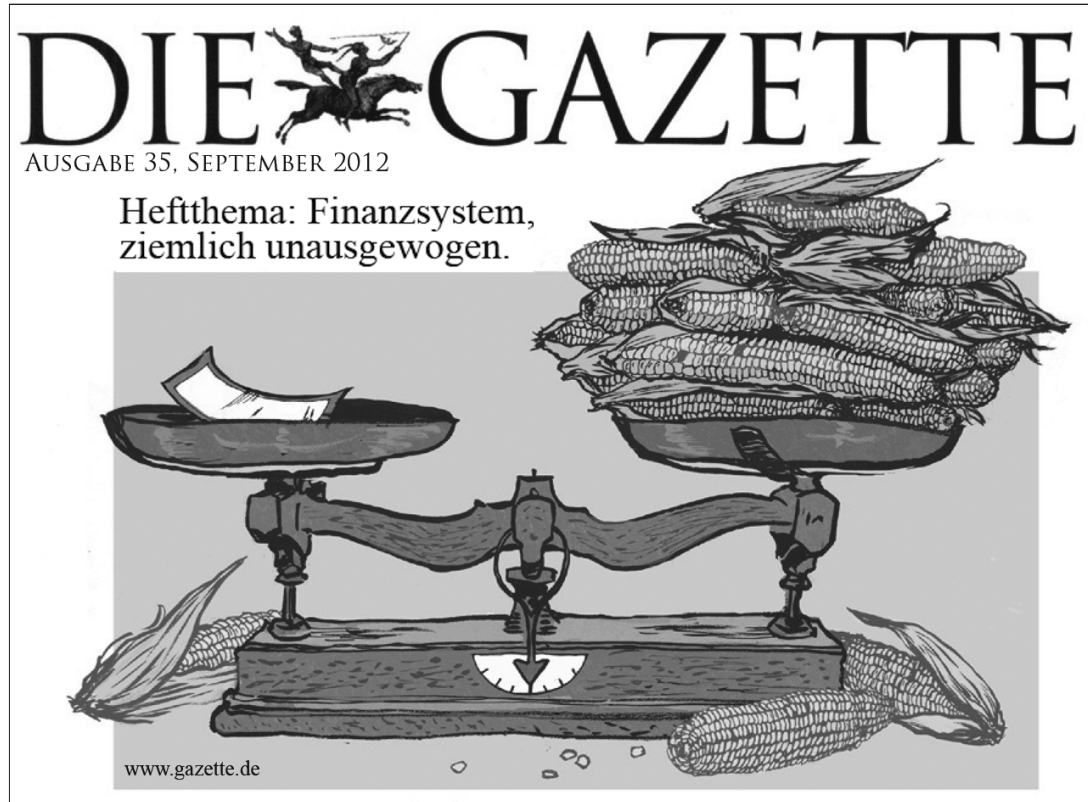

